\title{
Estimativa do Valor Energético dos Alimentos e Validação das Equações Propostas pelo NRC (2001) 1
}

\section{Vicente Ribeiro Rocha Júnior ${ }^{2}$, Sebastião de Campos Valadares Filho ${ }^{3}$, Álan Maia Borges ${ }^{4}$, Edenio Detmann ${ }^{5}$, Karla Alves Magalhães ${ }^{3}$, Rilene Ferreira Diniz Valadares ${ }^{3}$, Lúcio Carlos Gonçalves ${ }^{4}$, Paulo Roberto Cecon ${ }^{3}$}

\begin{abstract}
RESUMO - Neste trabalho objetivou-se estimar os valores energéticos dos alimentos, através das equações propostas pelo último NRC para gado leiteiro, utilizando como banco de dados as "Tabelas Brasileiras de Composição de Alimentos para Bovinos" e um experimento com ovinos, e validar essas equações a partir de observações referentes ao NDT e às frações digestíveis da fibra em detergente neutro (FDND), proteína bruta (PBD), extrato etéreo (EED) e carboidratos não fibrosos (CNFD), obtidas da literatura brasileira, a partir de experimentos in vivo, quando foram atendidas as exigências de mantença. O procedimento de validação dos parâmetros avaliados foi realizado por intermédio do ajuste de modelo de regressão linear simples dos valores preditos e dos observados. Os valores de NDT observados e preditos, tanto para os alimentos volumosos como para os concentrados, foram similares. Entretanto, quando se considerou apenas a estimativa da FDND de alimentos volumosos, verificou-se rejeição de ambas as hipóteses de nulidade, ou seja, tanto o intercepto quanto o coeficiente de inclinação foram diferentes de zero e um, respectivamente. Para a fração nitrogenada dos alimentos não foi constatada nenhuma diferença entre os parâmetros avaliados, havendo correspondência dos valores preditos aos observados. O EED de alimentos volumosos e concentrados, produzidos em condições tropicais, é subestimado pela equação utilizada pelo NRC, assim como os CNFD dos alimentos concentrados. Portanto, pode-se concluir que os valores de NDT obtidos a partir da equação proposta pelo NRC foram compatíveis com os dados observados de alimentos produzidos em condições brasileiras, todavia, sugere-se que sejam feitos alguns ajustes das equações que estimam as frações digestíveis da FDN, do EE e dos CNF.
\end{abstract}

Palavras-chave: fibra em detergente neutro digestível, NDT, ruminantes

\section{Estimative of Energy Value of Feed and Validation of Equations Proposed by NRC (2001)}

ABSTRACT - The objectives of this paper were estimate the energy values of feeds through equations proposed by NRC, from feed composition present in data base of "Brazilian Tables of Feed Composition for Cattle" and of a trial that used sheep, and validate those equations from observed values of TDN, digestible fractions of NDF (DNDF), crude protein (DCP), ether extract (DEE) and nonfiber carbohydrates (DNFC), that were obtained on Brazilian Scientific literature through in vivo experiments at maintenance level. The procedure to validate the parameters evaluated (TDN, DNDF, DCP, DEE, DNFC) was done by adjustment models of simple linear regression using predict and observed values. The observed and predict values of TDN values for forage and concentrate feeds were similar. However, when considering only the DNDF of forages estimative was verified rejection for both null hypothesis, because either intercept or coefficient of inclination were different of zero and 1, respectively. For the feed nitrogen fraction was not verified difference between the parameters evaluated. The DEE of forages and concentrate feeds was underestimated by the equations proposed by NRC, as well as the DNFC of concentrates feed. Thus, it can be concluded that the data obtained by the equations used by NRC to estimate the energy value of feed were similar with the observed data of Brazilian feeds, however, it is suggested that these equations should be adjusted to estimate the digestible fractions of NDF of forage, DEE and DNFC of brazilian feeds.

Key Words: digestible neutral detergent fiber, TDN, ruminants

\section{Introdução}

Em condições normais de alimentação, a energia é o nutriente que mais limita o desempenho dos ruminantes, merecendo, portanto, especial atenção dos nutricionistas no que diz respeito às exigências do animal e à sua disponibilidade nos alimentos. A quantidade total de energia contida em um alimento pode ser facilmente medida pela combustão deste em bomba calorimétrica; todavia, a variabilidade na

1 CNPq.

2 Universidade Estadual de Montes Claros - Av. Rui Braga s/n, Campus Universitário Darcy Ribeiro - Prédio da Reitoria, Vila Mauricéia,

Montes Claros (MG). E.mail: rochajuniorvr@hotmail.com

3 Universidade Federal de Viçosa - Av. PH Holfs, s/ n , Campus Universitário - CEP: 36570-000; Viçosa, MG.

4 Universidade Federal de MInas Gerais - Caixa Postal 567; CEP: 30123-970; Belo Horizonte, MG.

5 Universidade Estadual do Norte Fluminense - Campus dos Goitacazes, RJ. 
digestibilidade e no metabolismo entre os alimentos impede o uso da energia bruta para formulação de dietas ou comparação de alimentos (Weiss, 1993).

A dificuldade de se avaliar a disponibilidade energética e a importância de se conhecer o conteúdo de energia dos alimentos têm levado ao desenvolvimento de métodos para estimar o conteúdo de energia disponível. Equações de regressão têm várias limitações: o erro de predição pode ser alto, a equação é específica para uma dada população e, além disso, a maioria das equações tem sido derivada a partir de dados com forragens; poucas são disponíveis para estimar o valor energético dos concentrados (Weiss et al., 1992).

Muitos componentes químicos são relacionados à concentração de energia disponível de um alimento, sendo que os constituintes comumente avaliados são cinzas, extrato etéreo $(\mathrm{EE})$, proteína bruta $(\mathrm{PB})$, fibra em detergente neutro (FDN), fibra em detergente ácido (FDA), lignina, amido e frações nitrogenadas. Alguns destes componentes, especialmente gordura e PB, têm sido positivamente correlacionados ao NDT, enquanto que as frações fibrosas têm apresentado correlações negativas com a disponibilidade energética dos alimentos. A lignina, embora constitua somente pequena proporção dos alimentos ( 1 a 12\%), mostra alta correlação negativa com a digestibilidade (Weiss, 1998).

Modelos com múltiplos componentes, baseados na composição, podem ser empíricos ou mecanicísticos. Os modelos empíricos são essencialmente descrições diretas dos dados de observação, derivados por regressão de numerosas características dos alimentos com a digestibilidade, sendo específicos para determinada população. Os modelos mecanicísticos baseiamse em frações nutricionais uniformes, de digestibilidade constante, e devem ser independentes da população. Quer sejam empíricos ou mecanicísticos, os modelos com múltiplos compartimentos apresentam maior precisão que aqueles com compartimento único, haja vista que os primeiros consideram maior número de variáveis que os últimos (Weiss, 1998).

O NRC (2001) utilizou uma equação somativa que considera cada fração do alimento para estimar o NDT de mantença. No Brasil, essas equações deverão ser validadas antes de serem recomendadas para estimar o valor energético dos alimentos tropicais, principalmente dos volumosos, e especialmente no que diz respeito à digestibilidade da FDN.

Os objetivos deste trabalho foram estimar o NDT dos alimentos através das equações propostas pelo NRC (2001), a partir da composição de alimentos presente no banco de dados das Tabelas Brasileiras de Composição de Alimentos para Bovinos (Valadares Filho et al., 2002) e de um experimento com ovinos alimentados com diferentes alimentos e validar essas equações a partir de resultados de NDT e das frações digestíveis da FDN, da PB, do EE e dos CNF encontrados na literatura brasileira, e obtidos através de experimentos in vivo.

\section{Material e Métodos}

A partir da composição química dos alimentos avaliados no experimento com ovinos descrita por Rocha Júnior et al. (2002), foram estimados os valores de NDT para mantença, conforme as seguintes equações recomendadas pelo NRC (2001), que estima os teores de proteína bruta digestível (PBD), ácidos graxos digestíveis (AGD), fibra em detergente neutro livre de proteínas digestível (FDNpD) e carboidratos não fibrosos digestíveis (CNFD):

(1) PBD (para alimentos volumosos) $=\mathrm{PB} \times \mathrm{Exp}$ $(-1,2 \times(\mathrm{PIDA} / \mathrm{PB}) ;$ em que PIDA = proteína insolúvel em detergente ácido;

(2) $\mathrm{PBD}$ (para alimentos concentrados) = PB x [1- (0,4 x PIDA/PB)];

(3) $\mathrm{AGD}=(\mathrm{EE}-1) \times 100$;

(4) $\mathrm{CNFD}=0,98 \times \mathrm{CNF} \times \mathrm{PAF}$; em que $\mathrm{PAF}=$ fator de ajuste para processamento físico, considerando valores de 0,$92 ; 0,94 ; 1,04$; e 1,0 , respectivamente, para grão de sorgo, silagem de milho, farelo de cereais e demais alimentos.

(5) FDNpD = 0,75 (FDNp-L) x [1-(L/FDNp)0,667]; em que $\mathrm{L}=$ lignina;

Assim, para estimar os nutrientes digestíveis totais de mantença (NDTM), foi utilizada a equação: $\mathrm{NDTM}=\mathrm{PBD}+2,25 \mathrm{AGD}+\mathrm{FDNpD}+\mathrm{CNFD}-7$; em que o valor 7 se refere ao NDT fecal metabólico (NRC, 2001).

Já para os alimentos protéicos de origem animal, o NDT de mantença foi estimado pela seguinte equação: $\mathrm{NDTM}=\mathrm{PBD}+2,25 \mathrm{AGD}+0,98 \mathrm{CHOT}-7$, considerando-se $\mathrm{PBD}$ de 0,86 e 0,94 para as farinhas de sangue e peixe, respectivamente, e AGD = EE - 1 (NRC, 2001).

Também, a partir do banco de dados das Tabelas Brasileiras de Composição de Alimentos para Bovinos (Valadares Filho et al., 2002), foram estimados o NDT, utilizando-se as mesmas equações descritas 
acima. Os alimentos foram agrupados para as análises estatísticas em alimentos volumosos, alimentos concentrados e conjunto de alimentos.

A validação dos parâmetros avaliados (NDTM, FDNpD, PBD, EED e CNFD) foi realizada a partir de 45 observações de NDT (23 volumosos e 22 concentrados), 63 observações de FDNpD de alimentos volumosos, 71 observações de EED (54 volumosos e 17 concentrados) e 20 observações de CNFD. Essas observações também foram obtidas de Valadares Filho et al. (2002) a partir de experimentos in vivo realizados com bovinos, ovinos, caprinos e bubalinos, quando foram atendidas as exigências de mantença ou próximas a ela. A digestibilidade verdadeira da $\mathrm{PB}$, do EE e do CNF foi corrigida para excreção fecal metabólica para cada nutriente, segundo os valores utilizados por Weiss et al. (1992) e que foram adotados pelo NRC (2001). Desta forma, os valores estimados a partir das equações descritas em (1) e (2); (3); e (4) foram subtraídos em 2,7; 1,4; e 3,1 unidades percentuais para a PBD, o EED e os CNFD, respectivamente.

O procedimento de validação foi feito por intermédio do ajuste de modelo de regressão linear simples (Regressão 1) dos valores preditos e dos valores observados. As estimativas dos parâmetros de regressão foram testadas sob as seguintes hipóteses:
(1) $\mathrm{H} 0: \beta 0=0$
(2) $\mathrm{H} 0: \beta 1=1$
$\mathrm{Ha}: \beta 0 \neq 0$
На : $\beta 1 \neq 1$

No caso da rejeição da hipótese 1 e aceitação da 2, concluiu-se que há uma correspondência, com presença de vício constante, que corresponde à estimativa do intercepto. Já no caso de aceitação da primeira e rejeição da segunda hipótese de nulidade, é verificada a presença de vício global de estimação (VGE). No caso de não-rejeição de ambas as hipóteses de nulidade, concluiu-se que os valores preditos e observados são similares. Em situação contrária, nova equação de regressão foi traçada, suprimindose o parâmetro relativo ao intercepto (Regressão 2), de acordo com o seguinte modelo: $Y_{i}=\beta_{1} X_{i}+e_{i}$. Neste caso, certificou-se a presença de dois vícios, sendo considerado apenas o VGE.

O vício global de estimação (VGE) é estimado como:

$\operatorname{VGE}=\left(\beta_{1}-1\right) \times 100 ;$ em que: $\beta_{1}=$ estimativa do coeficiente angular da equação de regressão sem intercepto; e "1" = valor paramétrico para o coeficiente angular sob a pressuposição de H0 ser verdadeira. Adotou-se um $\alpha=0,05$ para os procedimentos estatísticos empregados.

\section{Resultados e Discussão}

A partir da composição química dos alimentos avaliados no experimento com ovinos descrita por Rocha Júnior et al. (2002), foram estimados os nutrientes digestíveis totais pela equação proposta pelo NRC (2001). Os alimentos foram agrupados em volumosos, concentrados e conjunto de alimentos para os procedimentos estatísticos adotados. Na Tabela 1, são apresentadas as médias, com os respectivos erros-padrão do NDT, preditas pelo NRC (2001) e observadas em experimento com ovinos, para cada categoria de alimentos. Na Tabela 2, estão presentes as estimativas dos parâmetros da regressão entre os valores preditos e observados.

Como pode ser observado, não houve diferenças significativas entre os valores de NDT observados e preditos, tanto para alimentos volumosos como para concentrados. Pelos bons coeficientes de determinação encontrados, com exceção para alimentos volumosos, supõe-se que a proposta do NRC (2001) a fim de estimar o valor energético dos alimentos possa ser utilizada na predição do NDT.

É importante considerar que os valores de NDT observados foram obtidos com ovinos alimentados sem restrição, enquanto as equações utilizadas foram desenvolvidas para estimar a energia disponível dos alimentos para bovinos, no nível de mantença. Portanto, apesar das estimativas dos coeficientes $\left(\hat{\beta}_{0}\right.$ e $\left.\hat{\beta}\right)$ das equações de regressão entre valores preditos e observados não terem diferido significativamente $(\mathrm{P}>0,05)$ de zero e um, respectivamente, sugere-se que estas equações sejam validadas a partir de uma nova base de dados, com maior número de observações.

Na publicação do NRC (1971), os valores de EL foram calculados a partir da energia digestível (ED), mas, a partir de 1979, a EL tem sido calculada a partir do NDT usando uma equação que faz ajuste da digestibilidade, em função do nível de ingestão, incorporando $8 \%$ de redução quando os animais são alimentados com elevados níveis de consumo (três vezes a mantença). O problema deste método é que todos os alimentos são descontados similarmente. Segundo Edionwen \& Owen (1989), quando a ingestão de matéria seca foi modificada, a digestibilidade não foi alterada igualmente para todos os nutrientes ou alimentos, sendo que dietas com alta digestibilidade na mantença apresentam maior taxa de queda com o nível de alimentação do que aquelas com menor digestibilidade. Tyrrell \& Moe (1972, 1974 e 1975) 
citados pelo NRC (2001), demonstraram que a taxa de declínio na digestibilidade com o nível de alimentação foi função da digestibilidade da dieta na mantença.

Assim, o NRC (2001) sugeriu um fator para corrigir o efeito do nível de consumo sobre a digestibilidade e, conseqüientemente, o valor energético dos alimentos, sendo este fator (FC) calculado pela seguinte equação:

$\mathrm{FC}=\mathrm{NDT}-[(0,18 \mathrm{NDT}-10,3) \mathrm{x}$ Nível de Ingestão]/NDT

O nível de ingestão é dado em termos dos múltiplos da exigência de energia líquida para mantença. Multiplicando-se o FC pelo teor de EDmantença, obtém-se a EDprodutiva, sendo posteriormente utilizadas equações para estimar a EMprodutiva e a ELprodutiva. Esse FC deve ser adotado apenas para dietas com mais de 60\% de NDT. Segundo Vandehaar (1998), a depressão na digestibilidade em função da taxa de consumo é insignificante para dietas com valores de NDT iguais ou inferiores a $60 \%$. Nesse sentido, deve-se ressaltar que o NDT das dietas do experimento com ovinos, a partir das quais foram determinados os valores de NDT dos alimentos, variou de 49,76 a $65,94 \%$, com $70 \%$ dos valores situando-se abaixo de $60 \%$, o que de certa forma credibiliza os resultados encontrados com a base de dados considerada.

Utilizando-se o banco de dados das Tabelas Brasileiras de Composição de Alimentos para Bovinos (Valadares Filho et al., 2002) foi também estimado o NDT dos alimentos no nível de mantença. A tentativa

Tabela 1 - Médias e respectivos erros-padrão do NDT predito pelo NRC (2001) e observado no experimento com ovinos Table 1 - Averages and standard errors of TDN predicted by NRC (2001) and observed from trial with sheep

\begin{tabular}{|c|c|c|c|c|}
\hline \multirow[t]{2}{*}{$\begin{array}{l}\text { Variável } \\
\text { Variable }\end{array}$} & \multicolumn{2}{|c|}{$\begin{array}{l}\text { Valores preditos } \\
\text { Predicted values }\end{array}$} & \multicolumn{2}{|c|}{$\begin{array}{c}\text { Valores observados } \\
\text { Observed values }\end{array}$} \\
\hline & $\begin{array}{l}\text { Média } \\
\text { Average }\end{array}$ & $\begin{array}{l}\text { Erro-padrão } \\
\text { Standard error }\end{array}$ & $\begin{array}{l}\text { Média } \\
\text { Average }\end{array}$ & $\begin{array}{l}\text { Erro-padrão } \\
\text { Standard error }\end{array}$ \\
\hline $\begin{array}{l}\text { NDTv(1) } \\
T D N f\end{array}$ & 57,14 & $\pm 3,54$ & 54,84 & $\pm 2,41$ \\
\hline $\begin{array}{l}\operatorname{NDTc}^{(2)} \\
T D N c\end{array}$ & 75,52 & $\pm 3,15$ & 77,93 & $\pm 3,42$ \\
\hline $\begin{array}{l}\text { NDTcj }^{3} \\
\text { TDNaf }\end{array}$ & 69,39 & $\pm 3,15$ & 70,23 & $\pm 3,55$ \\
\hline
\end{tabular}

${ }^{1}$ Alimentos volumosos; ${ }^{2}$ Alimentos concentrados; ${ }^{3}$ Conjunto de alimentos (volumosos + concentrados).

${ }^{1}$ Forages, ${ }^{2}$ Concentrate feeds, ${ }^{3}$ All feeds (forage + concentrate).

Tabela 2 - Estimativa dos coeficientes da regressão entre os valores de NDT observados e os preditos pela equação do NRC (2001)

Table 2 - Estimative of regression coefficients between observed TDN values and predicted by NRC (2001)

\begin{tabular}{|c|c|c|c|c|c|}
\hline \multirow[t]{3}{*}{$\begin{array}{l}\text { Variável } \\
\text { Variable }\end{array}$} & \multicolumn{4}{|c|}{$\begin{array}{l}\text { Regressão } \\
\text { Regression }^{1}\end{array}$} & \multirow{3}{*}{$\mathrm{R}^{2}$} \\
\hline & \multicolumn{2}{|c|}{$\begin{array}{c}\text { Intercepto }\left(\hat{\beta}_{0}\right) \\
\text { Intercept }\end{array}$} & \multicolumn{2}{|c|}{$\begin{array}{c}\text { Coeficiente de inclinação }(\hat{\beta}) \\
\text { Coefficient of inclination }\end{array}$} & \\
\hline & $\begin{array}{c}\text { Estimativa } \\
\text { Estimate }\end{array}$ & $\mathrm{P}^{(2)}$ & $\begin{array}{c}\text { Estimativa } \\
\text { Estimate }\end{array}$ & $\mathrm{P}^{(3)}$ & \\
\hline NDTr(4) & $-8,7278$ & 0,73 & 1,2011 & 0,66 & 0,67 \\
\hline $\begin{array}{l}T D N f \\
\operatorname{NDTC}^{(5)} \\
T D N c\end{array}$ & 7,6847 & 0,49 & 0,8705 & 0,35 & 0,89 \\
\hline $\begin{array}{l}\operatorname{NDTcj}^{(6)} \\
\text { TDNaf }\end{array}$ & 10,091 & 0,10 & 0,8444 & 0,08 & 0,90 \\
\hline
\end{tabular}

${ }^{1} \mathrm{Y}_{\mathrm{i}}=\hat{\beta}_{0}+\hat{\beta} \mathrm{X}_{\mathrm{i}} ;{ }^{2} \mathrm{H}_{\mathrm{o}}: \beta_{0}=0 ;{ }^{3} \mathrm{H}_{\mathrm{o}}: \beta_{1}=1 ;{ }^{4}$ Alimentos volumosos; ${ }^{5}$ Alimentos concentrados; ${ }^{6}$ Conjunto de alimentos (volumoso + concentrado).

${ }^{4}$ Forages, ${ }^{5}$ Concentrate feeds, ${ }^{6}$ All feeds (forage + concentrate). 
de validação destas estimativas se procedeu a partir de uma base de dados com maior número de valores observados obtidos da literatura nacional através de experimentos in vivo, sendo que na maioria foram atendidas as exigências de mantença ou próximas a ela. Como pode ser verificado nas Tabelas 3 e 4, os valores de NDT observados e preditos, tanto para volumosos como para concentrados, foram semelhantes $(\mathrm{P}>0,05)$, apesar do coeficiente de determinação da equação de regressão para alimentos volumosos ter sido baixo. Confirmando-se assim os resultados encontrados anteriormente, inclusive pela similaridade dos coeficientes de determinação das equações de regressão para cada categoria de alimentos. Portanto, pode-se concluir que a proposta do NRC
(2001) para estimar o valor energético dos alimentos foi compatível com os valores determinados em condições brasileiras.

Constam nas Tabelas 5 e 6 , respectivamente, as médias com os valores de erro padrão da fração digestível da FDN, predita e observada a partir do experimento com ovinos (Rocha Júnior et al., 2002), e as estimativas dos parâmetros da regressão entre estas variáveis. Para alimentos concentrados, a FDND, predita e observada, foi similar $(\mathrm{P}>0,05)$. Entretanto, quando se considerou apenas a estimativa da FDND de alimentos volumosos, verificou-se rejeição de ambas hipóteses de nulidade, ou seja, tanto o intercepto como o coeficiente de inclinação foram diferentes de zero e um, respectivamente, com um vício numérico de

Tabela 3 - Médias e erros-padrão do NDT predito pelo NRC (2001) e observado

Table 3 - Averages and standard errors of TDN values predicted by NRC (2001) and observed

\begin{tabular}{|c|c|c|c|c|}
\hline \multirow[t]{2}{*}{$\begin{array}{l}\text { Variável } \\
\text { Variable }\end{array}$} & \multicolumn{2}{|c|}{$\begin{array}{l}\text { Valores preditos } \\
\text { Predicted values }\end{array}$} & \multicolumn{2}{|c|}{$\begin{array}{c}\text { Valores observados } \\
\text { Observed values }\end{array}$} \\
\hline & $\begin{array}{l}\text { Média } \\
\text { Average }\end{array}$ & $\begin{array}{l}\text { Erro-padrão } \\
\text { Standard error }\end{array}$ & $\begin{array}{l}\text { Média } \\
\text { Average }\end{array}$ & $\begin{array}{l}\text { Erro-padrão } \\
\text { Standard error }\end{array}$ \\
\hline $\begin{array}{l}\text { NDTv(1) } \\
T D N f\end{array}$ & 53,31 & $\pm 1,42$ & 54,81 & $\pm 1,34$ \\
\hline $\begin{array}{l}\text { NDTd } \\
\text { TDNf }\end{array}$ & 73,97 & $\pm 2,51$ & 73,63 & $\pm 2,41$ \\
\hline NDTcj(3) & 63,41 & $\pm 2,10$ & 64,02 & $\pm 1,96$ \\
\hline TDNaf & & & & \\
\hline
\end{tabular}

${ }^{1}$ Alimentos volumosos; ${ }^{2}$ Alimentos concentrados; ${ }^{3}$ Conjunto de alimentos (volumoso + concentrado).

${ }^{1}$ Forages, ${ }^{2}$ Concentrate feeds, ${ }^{3}$ All feeds (forage + concentrate)

Tabela 4 - Estimativa dos coeficientes da regressão entre os valores do NDT observados e preditos pela equação do NRC (2001)

Table 4 - Estimate of regression coefficients between observed TDN values and predicted by NRC (2001)

\begin{tabular}{|c|c|c|c|c|c|}
\hline \multirow[t]{3}{*}{$\begin{array}{l}\text { Variável } \\
\text { Variable }\end{array}$} & \multicolumn{4}{|c|}{$\begin{array}{l}\text { Regressão }^{1} \\
\text { Regression }^{1}\end{array}$} & \multirow{3}{*}{$\mathrm{R}^{2}$} \\
\hline & \multicolumn{2}{|c|}{$\begin{array}{c}\text { Intercepto }\left(\hat{\boldsymbol{\beta}}_{0}\right) \\
\text { Intercept }\end{array}$} & \multicolumn{2}{|c|}{$\begin{array}{l}\text { Coeficiente de inclinação }(\hat{\beta}) \\
\text { Coefficient of inclination }\end{array}$} & \\
\hline & $\begin{array}{l}\text { Estimativa } \\
\text { Estimate }\end{array}$ & $\mathrm{P}^{(2)}$ & $\begin{array}{c}\text { Estimativa } \\
\text { Estimate }\end{array}$ & $\mathrm{P}^{(3)}$ & \\
\hline $\begin{array}{l}\text { NDTv(4) } \\
T D N f\end{array}$ & 6,8991 & 0,37 & 0,8468 & 0,28 & 0,64 \\
\hline $\begin{array}{l}\operatorname{NDTd}^{(5)} \\
T D N c\end{array}$ & 0,6233 & 0,90 & 0,9961 & 0,95 & 0,92 \\
\hline $\begin{array}{l}\text { NDTcj(6) } \\
\text { TDNaf }\end{array}$ & $-2,6184$ & 0,37 & 1,0315 & 0,48 & 0,93 \\
\hline
\end{tabular}


ROCHA JÜNIOR et al.

Tabela 5 - Médias e respectivos erros-padrão da fração digestível da FDN (FDND) predita pelo NRC (2001) e observada no experimento com ovinos

Table 5 - Averages and standard errors of digestible fraction of NDF (NDFD) predicted by NRC (2001) and observed in the trial with sheep

\begin{tabular}{|c|c|c|c|c|}
\hline \multirow[t]{2}{*}{$\begin{array}{l}\text { Variável } \\
\text { Variable }\end{array}$} & \multicolumn{2}{|c|}{$\begin{array}{l}\text { Valores preditos } \\
\text { Predicted values }\end{array}$} & \multicolumn{2}{|c|}{$\begin{array}{l}\text { Valores observados } \\
\text { Observed values }\end{array}$} \\
\hline & $\begin{array}{l}\text { Média } \\
\text { Average }\end{array}$ & $\begin{array}{l}\text { Erro-padrão } \\
\text { Standard error }\end{array}$ & $\begin{array}{l}\text { Média } \\
\text { Average }\end{array}$ & $\begin{array}{l}\text { Erro-padrão } \\
\text { Standard error }\end{array}$ \\
\hline $\mathrm{FDNDv}^{1}(D N D F v)$ & 35,84 & $\pm 3,94$ & 29,81 & $\pm 5,84$ \\
\hline $\mathrm{FDNDc}^{2}(D N D F c)$ & 17,81 & $\pm 3,64$ & 15,20 & $\pm 3,84$ \\
\hline FDNDcj $^{3}$ (DNDFaf) & 23,82 & $\pm 3,39$ & 20,07 & $\pm 3,54$ \\
\hline
\end{tabular}

${ }^{1}$ Alimentos volumosos; ${ }^{2}$ Alimentos concentrados; ${ }^{3}$ Conjunto de alimentos (volumoso + concentrado).

${ }^{1}$ Forages, ${ }^{2}$ Concentrate feeds, ${ }^{3}$ All feeds (forage + concentrate)

Tabela 6 - Estimativa dos coeficientes da regressão entre os valores da fração de FDN digestível (FDND) observada e predita pela equação do NRC (2001)

Table 6 - Estimate of regression coefficients between digestible fraction of observed NDF (NDFD) values and predicted by NRC (2001)

\begin{tabular}{|c|c|c|c|c|c|c|c|c|c|c|}
\hline \multirow[t]{3}{*}{$\begin{array}{l}\text { Variável } \\
\text { Variable }\end{array}$} & \multicolumn{5}{|c|}{$\begin{array}{l}\text { Regressão }{ }^{1} \\
\text { Regression }\end{array}$} & & \multicolumn{4}{|c|}{$\begin{array}{l}\text { Regressão } \\
\text { Regression }^{2}\end{array}$} \\
\hline & \multicolumn{2}{|c|}{$\begin{array}{c}\text { Intercepto }\left(\hat{\beta_{0}}\right) \\
\text { Intercept }\end{array}$} & \multicolumn{2}{|c|}{$\begin{array}{l}\text { Coeficiente de inclinação }\left(\hat{\boldsymbol{\beta}}_{1}\right) \\
\text { Coefficient of inclination } \\
\end{array}$} & & \multirow{2}{*}{$\begin{array}{c}\text { Vício } \\
\text { constante } \\
\text { Constant bias }\end{array}$} & \multicolumn{2}{|c|}{$\begin{array}{l}\text { Coeficiente de inclinação }\left(\hat{\beta}_{1}\right) \\
\text { Coefficient of inclination } \\
\end{array}$} & \multicolumn{2}{|c|}{$\begin{array}{l}\mathrm{R}^{2} \text { Vício } \\
-\quad \text { global }^{6}\end{array}$} \\
\hline & $\begin{array}{l}\text { Estimativa } \\
\text { Estimate }\end{array}$ & $\mathrm{P}^{3}$ & $\begin{array}{l}\text { Estimativa } \\
\text { Estimate }\end{array}$ & $\mathrm{P}^{4}$ & & & $\begin{array}{l}\text { Estimativa } \\
\text { Estimate }\end{array}$ & $\mathrm{P}^{4}$ & & bal bias \\
\hline $\begin{array}{l}\text { FDNDv }{ }^{7} \\
D N D F v^{7}\end{array}$ & 17,3318 & 0,02 & 0,6209 & 0,05 & 0,85 & - & 1,1087 & 0,36 & 0,96 & 10,87 \\
\hline $\begin{array}{l}\text { FDNDc }^{8} \\
D N D F c^{8}\end{array}$ & 4,1765 & 0,06 & 0,8975 & 0,32 & 0,89 & - & - & - & - & - \\
\hline $\begin{array}{l}\text { FDNDcj }^{9} \\
\text { DNDFaf }\end{array}$ & 5,7944 & 0,01 & 0,8985 & 0,24 & 0,88 & 5,79 & - & - & - & - \\
\hline
\end{tabular}

${ }^{1} Y_{i}=\hat{\beta_{0}}+X_{i} ;{ }^{2} Y_{i}=\hat{\beta}_{1} X_{i} ;{ }^{3} H_{0}: \beta_{0}=0 ; 4 / H_{0}: \beta_{1}=1 ;{ }^{5}$ Em pontos percentuais; ${ }^{6}$ Em porcentagem; ${ }^{7}$ Alimentos volumosos; ${ }^{8}$ Alimentos concentrados; ${ }^{9}$ Conjunto de alimentos (volumoso + concentrado).

${ }^{5}$ Percentage points; ${ }^{6}$ In percentage; ${ }^{7}$ Forage, ${ }^{8}$ Concentrate feeds; ${ }^{9}$ All feeds (forage + concentrate).

10,87\%. Quando nova equação de regressão foi traçada, suprimindo-se o parâmetro relativo ao intercepto (Regressão 2), o coeficiente de inclinação não diferiu estatisticamente de um $(\mathrm{P}>0,05)$, o que é incoerente com o resultado apresentado pela primeira regressão (Regressão 1). Infelizmente a análise gráfica dos resíduos ordinários não foi conclusiva, devido ao pequeno número de observações referente a alimentos volumosos (6). Assim, a estimação da FDND pelo NRC (2001), para alimentos volumosos, produzidos em condições tropicais, deve ser testada, utilizando-se maior número de observações.

Utilizando-se a base de dados das Tabelas Brasileiras de Composição de Alimentos para Bovinos (Valadares Filho et al., 2002), a FDND de alimentos volumosos, estimada pelo NRC (2001), foi novamente testada. Nas Tabelas 7 e 8 apresentam-se as médias da FDND de alimentos volumosos e as estimativas dos parâmetros da regressão entre valores preditos e observados. A dispersão dos pontos em torno da reta "Y = X" pode ser visualizada na Figura 1. Como pode ser visto, a análise de regressão teve o mesmo comportamento verificado com a análise realizada a partir dos valores observados de FDND do experimento com ovinos. Tanto o intercepto como o coeficiente de inclinação diferiram $(\mathrm{P}<0,05)$ de zero e um, respectivamente. Embora a análise da equação de regressão sem intercepto (Regressão 2) não tenha constatado diferença $(\mathrm{P}>0,05)$ para o parâmetro avaliado (b1), a análise gráfica de resíduos (Figuras 2 e 3) 
Tabela 7 - Médias e respectivos erros-padrão da fração digestível da FDN (FDND) predita pelo NRC (2001) e observada Table 7 - Averages and standard errors of digestible fraction of observed NDF (NDFD) and predicted by NRC (2001)

\begin{tabular}{|c|c|c|c|c|}
\hline \multirow[t]{2}{*}{$\begin{array}{l}\text { Variável } \\
\text { Variable }\end{array}$} & \multicolumn{2}{|c|}{$\begin{array}{l}\text { Valores preditos } \\
\text { Predicted values }\end{array}$} & \multicolumn{2}{|c|}{$\begin{array}{c}\text { Valores observados } \\
\text { Observed values }\end{array}$} \\
\hline & $\begin{array}{l}\text { Média } \\
\text { Average }\end{array}$ & $\begin{array}{l}\text { Erro-padrão } \\
\text { Standard error }\end{array}$ & $\begin{array}{l}\text { Média } \\
\text { Average }\end{array}$ & $\begin{array}{l}\text { Erro-padrão } \\
\text { Standard error }\end{array}$ \\
\hline $\begin{array}{l}\text { FDNDv }{ }^{1} \\
D N D F v^{1}\end{array}$ & 35,66 & $\pm 0,82$ & 35,36 & $\pm 1,08$ \\
\hline
\end{tabular}

${ }^{1}$ Alimentos volumosos (forages).

Tabela 8 - Estimativa dos coeficientes da regressão entre os valores de FDND observados e preditos pela equação do NRC (2001)

Table 8 - Estimate of regression coefficients between digestible fraction of observed NDF (NDFD) values and predicted by NRC (2001)

\begin{tabular}{|c|c|c|c|c|c|c|c|c|c|}
\hline \multirow[t]{3}{*}{$\begin{array}{l}\text { Variável } \\
\text { Variable }\end{array}$} & \multicolumn{6}{|c|}{$\begin{array}{l}\text { Regressão } \\
\text { Regression }^{1}\end{array}$} & \multicolumn{3}{|c|}{$\begin{array}{l}\text { Regressão }^{2} \\
\text { Regression }^{2}\end{array}$} \\
\hline & \multicolumn{2}{|c|}{$\begin{array}{c}\text { Intercepto }\left(\hat{\beta_{0}}\right) \\
\text { Intercept }\end{array}$} & \multicolumn{2}{|c|}{$\begin{array}{l}\text { Coeficiente de inclinação }\left(\hat{\beta}_{1}\right) \\
\text { Coefficient of inclination }\end{array}$} & \multirow{2}{*}{\multicolumn{2}{|c|}{$\begin{array}{l}\mathrm{R}^{2} \text { Vício } \\
\text { constante } \\
\text { Constant bias }\end{array}$}} & $\begin{array}{r}\text { Coeficiente de incl } \\
\text { Coefficient of inc }\end{array}$ & $\begin{array}{l}\text { ação }\left(\hat{\beta}_{1}\right) \\
\text { lation }\end{array}$ & $\begin{array}{l}\mathrm{R}^{2} \text { Vício } \\
\text { global }^{6}\end{array}$ \\
\hline & $\begin{array}{c}\text { Estimativa } \\
\text { Estimate }\end{array}$ & $\mathrm{P}^{3}$ & $\begin{array}{c}\text { Estimativa } \\
\text { Estimate }\end{array}$ & $\mathrm{P}^{4}$ & & & $\begin{array}{c}\text { Estimativa } \\
\text { Estimate }\end{array}$ & $\mathrm{P}^{4}$ & Global bias 6 \\
\hline $\begin{array}{l}\text { FDNDv }{ }^{7} \\
D N D F v^{7}\end{array}$ & 15,1031 & $<0,01$ & 0,5815 & $<0,01$ & 0,59 & - & 0,9853 & 0,44 & $-1,47$ \\
\hline
\end{tabular}

${ }^{1} \mathrm{Y}_{\mathrm{i}}=\hat{\beta_{0}}+\hat{\beta_{1}} \mathrm{X}_{\mathrm{i}} ;{ }^{2} \mathrm{Y}_{\mathrm{i}}=\hat{\boldsymbol{\beta}_{1}} \mathrm{X}_{\mathrm{i}} ;{ }^{3} \mathrm{H}_{0}: \beta_{0}=0 ;{ }^{4} \mathrm{H}_{0}: \beta_{1}=1 ;{ }^{5} \mathrm{Em}$ pontos percentuais; ${ }^{6} \mathrm{Em}$ porcentagem; ${ }^{7} \mathrm{Alimentos} \mathrm{volumosos.}$

5 Percentage points; ${ }^{6}$ In percentage; ${ }^{7}$ Forage.

permitiu concluir que a FDND de alimentos volumosos determinada em condições brasileiras é subestimada pela equação utilizada pelo NRC (2001), havendo um vício numérico de $-1,47 \%$. Contudo, observa-se que a média dos valores preditos é numericamente superior à dos valores observados, o que é facilmente explicável pelas diferenças nos valores de erro padrão das respectivas médias. Portanto, um ajuste desta equação deve ser feito considerando-se resultados nacionais.

Goering \& Van Soest (1970), citados por Weiss (1993), desenvolveram uma equação somativa para estimar a digestibilidade da matéria seca, levando em consideração a fração solúvel em detergente neutro e a FDND. A limitação desta equação é que a digestibilidade da FDN deve ser conhecida, e como esta não é constante entre os alimentos torna-se necessário o uso de diferentes valores. Assim, Conrad et al. (1984) propuseram uma equação somativa para predizer a EL de lactação de alguns alimentos a partir da composição química, considerando-se os mesmos princípios delineados por Goering \& Van Soest (1970). O novo aspecto desse modelo foi o uso de uma relação de área de superfície para estimar a proporção de FDN indisponível, sendo que, para isso, foram feitas correções para o teor de lignina do alimento. Segundo Weiss et al. (1992), a maioria, se não todos os alimentos, contém nitrogênio insolúvel em detergente neutro (NIDN), mas a proporção de FDN que é NIDN varia entre os alimentos. Estes autores modificaram o modelo de Conrad et al. (1984), ajustando a FDN para o nitrogênio.

Tentou-se ajustar os coeficientes da equação, por intermédio de regressão não linear: (5) $\mathrm{FDNpD}=0,75$ (FDNp - L) x [1- (L/FDNp)0,667], utilizando os valores observados de FDND encontrados na literatura brasileira. Os valores que minimizaram a soma de quadrados dos desvios, com o menor número de iterações, foram 0,6232 e 1,2258, respectivamente, para os coeficientes a e b (Tabela 9), obtendo-se a seguinte equação: 


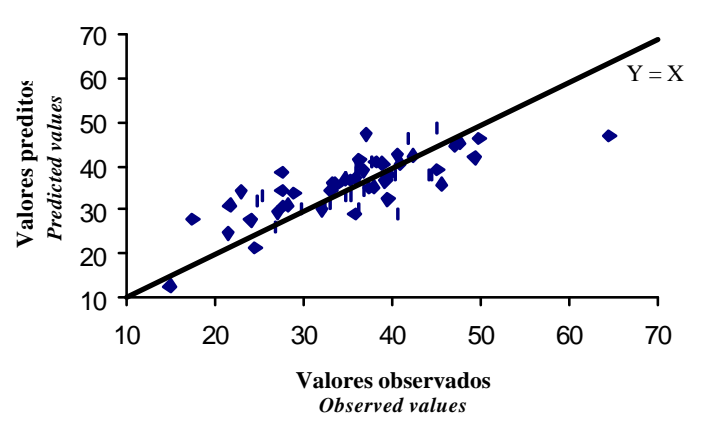

Figura 1 - Relação entre os valores de FDND observados e preditos pela equação do NRC (2001) para alimentos volumosos.

Figure 1 - Relation between values of NDFD observed and predicted by equation of NRC (2001) for forages.

Resíduos ordinários (c/ intercepto)

Ordinary residues (with intercept)

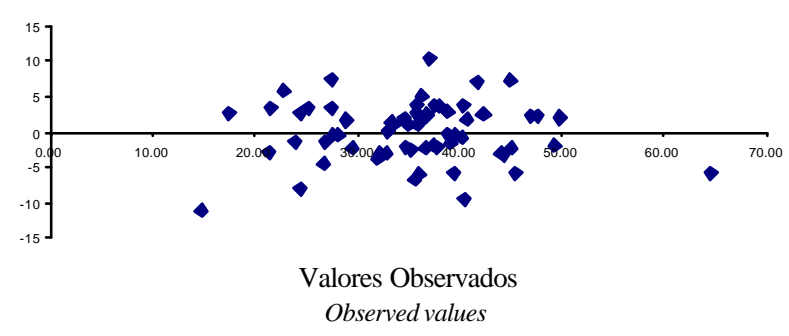

Figura 2 - Distribuição de resíduos ordinários dos valores FDND para equação de regressão com intercepto, em função dos valores observados.

Figure 2 - Distribution of ordinary residues of NDFD to regression equation with intercept, in function of observed values.

Resíduos Ordinários (s/ intercepto)

Ordinary residues (out intercept)

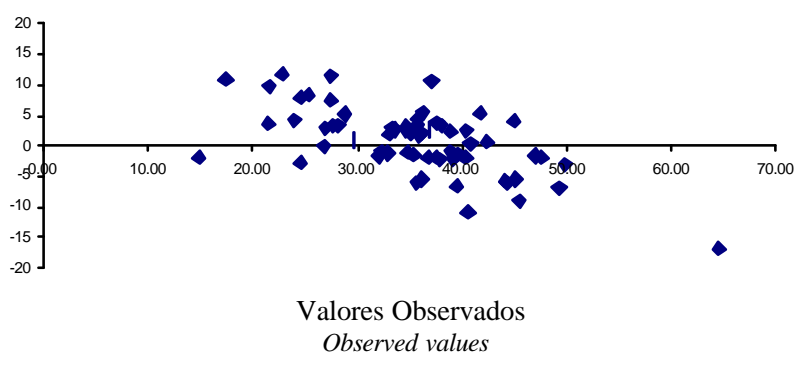

Figura 6 - Distribuição dos resíduos ordinários dos valores de FDND para equação de regressão sem intercepto, em função dos valores observados.

Figure 6 - Distribution of ordinary residues of NDFD to regression equation with out intercept, in function of observed values.
FDNpD = 0,6232 (FDNp - L) x [1- (L/FDNp)1,2258]

Por intermédio de modelo de regressão linear simples, os valores preditos a partir desta equação foram confrontados com os observados. Os resultados desta análise (Tabela 10) mostraram comportamento idêntico aos verificados anteriormente, não sendo possível o ajuste dessa equação para estimar a energia disponível da FDN de volumosos produzidos em condições tropicais. Entretanto, o vício numérico foi reduzido de $-1,47$ para $-0,77 \%$.

Como pode ser observado na Tabela 9, o valor de 0,75 para o coeficiente de digestibilidade da FDN potencialmente disponível proposto pelo NRC (2001) ficou fora do intervalo de confiança (a) da equação ajustada. Por outro lado, embora o coeficiente que relaciona a área de superfície entre lignina e FDN $(0,667)$ esteja contido no intervalo de confiança (b), a grande amplitude deste intervalo pode indicar que talvez a relação lignina/FDN não seja tão precisa para estimar a FDN disponível, devido à variabilidade da matriz fibrosa entre forrageiras tropicais.

A lignina tem sido reconhecida como o principal componente químico, afetando a digestibilidade da parede celular, cujo efeito direto tem sido explicado por diferentes hipóteses, tais como: o seu efeito tóxico aos microrganismos fibrolíticos, a limitação da ação das enzimas fibrolíticas resultante da deposição dos polímeros de lignina com a maturidade da planta, e o impedimento físico causado pela ligação polissacarídeolignina, o que limitaria o acesso das enzimas.

Na Tabela 11, são apresentadas as médias com os respectivos erros-padrão das frações digestíveis da proteína bruta, do extrato etéreo e dos carboidratos não fibrosos, preditas e observadas.

Os valores preditos de PBD, EED e CNFD, estimados a partir das características químicas e mostrados na Tabela 11, foram corrigidos para excreção fecal metabólica de cada nutriente, segundo valores propostos por Weiss et al. (1992) e que foram adotados pelo NRC (2001).

Constam na Tabela 12 as estimativas dos parâmetros das regressões para as seguintes variáveis: PBD, EED e CNFD. Para a fração nitrogenada dos alimentos, não foi constatada nenhuma diferença significativa $(\mathrm{P}>0,05)$ entre os parâmetros avaliados, havendo boa correspondência entre valores preditos e observados. Já para o EED, foi verificada a presença de vício constante nas estimativas, que subestimaram em 1,42 e 1,99 unidades percentuais a digestibilidade aparente do EE de alimentos volumosos 
Tabela 9 - Parâmetros estimados pela regressão não linear com respectivos desvios-padrão e intervalos de confiança Table 9 - Parameters estimated by non-linear regression with respective standard deviations and confidence intervals

\begin{tabular}{lccc}
\hline $\begin{array}{l}\text { Parâmetros } \\
\text { Parameters }\end{array}$ & $\begin{array}{c}\text { Estimativa } \\
\text { Estimate }\end{array}$ & $\begin{array}{c}\text { Desvio-padrão } \\
\text { Standard deviation }\end{array}$ & \multicolumn{2}{c}{$\begin{array}{c}\text { Intervalo de confiança } \\
\text { Confidence interval }\end{array}$} \\
\cline { 2 - 4 } $\begin{array}{l}\text { Coeficiente (a) } \\
\text { Coefficient }\end{array}$ & 0,6232 & $\begin{array}{c}\text { Limite inferior } \\
\text { Lower limit }\end{array}$ & 0,7215 \\
$\begin{array}{l}\text { Coeficiente (b) } \\
\text { Coefficient }\end{array}$ & 1,2258 & $\pm 0,05$ & 0,5250 \\
\hline
\end{tabular}

${ }_{1}^{1}$ Referente ao coeficiente de digestibilidade da FDN potencialmente disponível.

${ }^{2}$ Referente a relação de área de superfície entre lignina e FDN.

${ }^{1}$ Refering to digestibility coefficient of potentially available NDF.

${ }^{2}$ Refering to relation of surface area between lignin and NDF.

Tabela 10 - Estimativa dos coeficientes da regressão entre os valores de FDND observados e preditos pela equação do NRC (2001) ajustada

Table 10 - Estimate of regression coefficients between digestible fraction of observed NDF (NDFD) values and predicted by adjusted equation of NRC (2001)

\begin{tabular}{|c|c|c|c|c|c|c|c|c|c|}
\hline \multirow[t]{3}{*}{$\begin{array}{l}\text { Variável } \\
\text { Variable }\end{array}$} & \multicolumn{5}{|c|}{$\begin{array}{l}\text { Regressão } \\
\text { Regression }^{1}\end{array}$} & & \multicolumn{3}{|c|}{$\begin{array}{l}\text { Regressão } \\
\text { Regression }\end{array}$} \\
\hline & \multicolumn{2}{|c|}{$\begin{array}{l}\text { Intercepto }\left(\hat{\beta_{0}}\right) \\
\text { Intercept }\end{array}$} & \multicolumn{2}{|c|}{$\begin{array}{l}\text { Coeficiente de inclinação }\left(\hat{\beta}_{1}\right) \\
\text { Coefficient of inclination }\end{array}$} & \multirow{2}{*}{\multicolumn{2}{|c|}{$\begin{array}{l}\mathrm{R}^{2} \text { Vício } \\
\text { constante } \\
\text { Constant bias }\end{array}$}} & \multicolumn{2}{|c|}{$\begin{array}{l}\text { Coeficiente de inclinação }\left(\hat{\boldsymbol{\beta}}_{1}\right) \\
\text { Coefficient of inclination }\end{array}$} & \multirow{2}{*}{$\begin{array}{l}\mathrm{R}^{2} \begin{array}{c}\text { Vício } \\
\text { global }^{6}\end{array} \\
\text { Global bias }^{6}\end{array}$} \\
\hline & $\begin{array}{l}\text { Estimativa } \\
\text { Estimate }\end{array}$ & $\mathrm{P}^{3}$ & $\begin{array}{l}\text { Estimativa } \\
\text { Estimate }\end{array}$ & $\mathrm{P}^{4}$ & & & $\begin{array}{l}\text { Estimativa } \\
\text { Estimate }\end{array}$ & $\mathrm{P}^{4}$ & \\
\hline $\begin{array}{l}\text { FDNDv }{ }^{7} \\
D N D F v^{7}\end{array}$ & 15,0424 & $<0,01$ & 0,5812 & $<0,01$ & & 0,61 & 0,9923 & 0,66 & $-0,77$ \\
\hline
\end{tabular}

${ }^{1} \mathrm{Yi}=\hat{\beta_{0}}+\hat{\beta}_{1} \mathrm{X}_{\mathrm{i}} ;{ }^{2} \mathrm{Y}_{\mathrm{i}}=\hat{\beta}_{1} \mathrm{X}_{\mathrm{i}} ;{ }^{3} \mathrm{H}_{0}: \beta_{0}=0 ;{ }^{4} \mathrm{H}_{0}: \beta_{1}=1 ;{ }^{5} \mathrm{Em}$ pontos percentuais; ${ }^{6} \mathrm{Em}$ porcentagem; ${ }^{7}$ Alimentos volumosos.

${ }^{5}$ Percentage points; ${ }^{6}$ In percentage; ${ }^{7}$ Forage.

e concentrados, respectivamente. Quando foi considerado o conjunto de alimentos, o coeficiente de inclinação diferiu de um $(\mathrm{P}<0,05)$, sendo a digestibilidade desse nutriente subestimada em $29,57 \%$.

Muitos alimentos contêm entre 0 e $5 \%$ de EE. Todavia, os ácidos graxos agem como uma fração uniforme, mas a digestibilidade do $\mathrm{EE}$ varia entre os alimentos, especialmente quando forragens são comparadas com alimentos concentrados. A fração EE dos alimentos consiste de mistura heterogênea de substâncias pouco solúveis em água e solúveis em solventes orgânicos, incluindo compostos de alta digestibilidade (ácidos graxos), além de componentes com quase nenhuma energia digestível. Dessa forma, equações baseadas no EE não são acuradas para estimar a disponibilidade energética de uma população variada de alimentos (Weiss, 1993). Assim, para melhorar a acurácia da estimativa da energia disponível do EE, Weiss et al. (1992) recomendam a substituição do EE pelos ácidos graxos. Entretanto, o pequeno número de informações referentes à composição de ácidos graxos dos alimentos e a dificuldade de se avaliar rotineiramente os teores destes componentes dificultam a adoção dessa idéia na predição do conteúdo de energia disponível.

Com relação aos CNFD, observou-se que a proposta do NRC (2001) para estimar a fração digestível deste componente, subestima a digestibilidade total dos alimentos concentrados em 10,21\% (Tabela 12). Já para os volumosos, constatou-se similaridade $(\mathrm{P}>0,05)$ entre valores preditos e observados. Para o conjunto de alimentos, verificou-se um vício numérico de $-5,70 \%$.

Segundo Conrad et al. (1984), assim como cinzas, lignina, $\mathrm{PB}$ e ácidos graxos, os $\mathrm{CNF}$ agem como uma 
Tabela 11 - Médias e respectivos erros-padrão das frações digestíveis da PB (PBD), do EE (EED) e dos CNF (CNFD) preditas pelo NRC (2001) e observadas

Table 11 - Averages and standard errors of digestible fractions of CP (DCO), EE (DEE) and NFC (DNFC) predicted by NRC (2001) and observed

\begin{tabular}{|c|c|c|c|c|}
\hline \multirow[t]{2}{*}{$\begin{array}{l}\text { Variável } \\
\text { Variable }\end{array}$} & \multicolumn{2}{|c|}{$\begin{array}{l}\text { Valores preditos } \\
\text { Predicted values }\end{array}$} & \multicolumn{2}{|c|}{$\begin{array}{l}\text { Valores observados } \\
\text { Observed values }\end{array}$} \\
\hline & $\begin{array}{l}\text { Média } \\
\text { Average }\end{array}$ & $\begin{array}{l}\text { Erro-padrão } \\
\text { Standard error } \\
\end{array}$ & $\begin{array}{c}\text { Média } \\
\text { Average } \\
\end{array}$ & $\begin{array}{c}\text { Erro-padrão } \\
\text { Standard error }\end{array}$ \\
\hline $\mathrm{PBDv}^{1}(D C P f)$ & 5,76 & $\pm 0,44$ & 6,52 & $\pm 0,44$ \\
\hline $\operatorname{PBDc}^{2}(D C P c)$ & 19,05 & $\pm 3,95$ & 17,73 & $\pm 3,92$ \\
\hline $\operatorname{PBDcj}^{3}(D C P a f)$ & 9,47 & $\pm 1,34$ & 9,65 & $\pm 1,28$ \\
\hline $\operatorname{EEDv}^{1}(D E E f)$ & 0,56 & $\pm 0,15$ & 1,84 & $\pm 0,13$ \\
\hline $\operatorname{EEDc}^{2}(D E E c)$ & 2,14 & $\pm 1,23$ & 4,25 & $\pm 1,27$ \\
\hline $\operatorname{EEDcj}^{3}($ DEEal $)$ & 0,94 & $\pm 0,32$ & 2,41 & $\pm 0,34$ \\
\hline $\mathrm{CNFDv}^{1}(D N F C f)$ & 23,77 & $\pm 5,33$ & 21,13 & $\pm 4,98$ \\
\hline $\mathrm{CNFDc}^{2}(D N F C c)$ & 31,86 & $\pm 6,00$ & 35,87 & $\pm 6,50$ \\
\hline $\mathrm{CNFDcj}^{3}$ (DNFCaf) & 28,22 & $\pm 4,08$ & 29,23 & $\pm 4,45$ \\
\hline
\end{tabular}

${ }^{1}$ Alimentos volumosos; ${ }^{2}$ Alimentos concentrados; ${ }^{3}$ Conjunto de alimentos (volumoso + concentrado).

${ }^{1}$ Forage; ${ }^{2}$ Concentrate feeds; ${ }^{3}$ All feeds (forage + concentrate).

Tabela 12 - Estimativa dos coeficientes da regressão entre os valores observados e preditos pela equação do NRC (2001), para PBD, EED e CNFD

Table 12 - Estimate of regression coefficients among observed and predicted values by NRC (2001) for digestible fractions of CP (DCO), EE (DEE) and NFC (DNFC)

\begin{tabular}{|c|c|c|c|c|c|c|c|c|c|c|}
\hline \multirow[t]{3}{*}{$\begin{array}{l}\text { Variável } \\
\text { Variable }\end{array}$} & \multicolumn{4}{|c|}{$\begin{array}{l}\text { Regressão } \\
\text { Regression }^{1}\end{array}$} & & \multirow{3}{*}{$\begin{array}{c}\text { Vício } \\
\text { constante } \\
\text { Constant bias }\end{array}$} & \multicolumn{3}{|c|}{$\begin{array}{l}\text { Regressão } \\
\text { Regression }^{2}\end{array}$} & \multirow[b]{2}{*}{$\begin{array}{l}\text { Vício } \\
\text { global }^{6}\end{array}$} \\
\hline & \multicolumn{2}{|c|}{$\begin{array}{l}\text { Intercepto }\left(\hat{\beta_{0}}\right) \\
\text { Intercept }\end{array}$} & \multicolumn{2}{|c|}{$\begin{array}{l}\text { Coeficiente de inclinação }\left(\hat{\boldsymbol{\beta}}_{1}\right) \\
\text { Coefficient of inclination }\end{array}$} & & & \multicolumn{2}{|c|}{$\begin{array}{l}\text { Coeficiente de inclinação ( } \hat{\beta}_{1} \\
\text { Coefficient of inclination }\end{array}$} & \multirow{2}{*}{$\begin{array}{l}\mathrm{R}^{2} \\
- \\
\text { Glo }\end{array}$} & \\
\hline & $\begin{array}{c}\text { Estimativa } \\
\text { Estimate }\end{array}$ & $\mathrm{P}^{3}$ & $\begin{array}{c}\text { Estimativa } \\
\text { Estimate } \\
\end{array}$ & $\mathrm{P}^{4}$ & & & $\begin{array}{c}\text { Estimativa } \\
\text { Estimate } \\
\end{array}$ & $\mathrm{P}^{4}$ & & bal bias ${ }^{6}$ \\
\hline $\begin{array}{l}\mathrm{PBDv}^{7} \\
D C P^{7}\end{array}$ & $-0,3178$ & 0,34 & 0,9324 & 0,15 & 0,90 & - & - & - & - & - \\
\hline $\begin{array}{l}\operatorname{PBDc}^{8} \\
D C P c^{8}\end{array}$ & 1,4244 & 0,18 & 0,9939 & 0,89 & 0,97 & - & - & - & - & - \\
\hline $\begin{array}{l}\text { PBDcj }^{9} \\
\text { DCPaf }^{9}\end{array}$ & $-0,5106$ & 0,12 & 1,0344 & 0,14 & 0,97 & - & - & - & - & - \\
\hline $\begin{array}{l}\mathrm{EEDv}^{7} \\
D E E f^{7}\end{array}$ & $-1,4252$ & $<0,01$ & 1,0802 & 0,21 & 0,85 & $-1,43$ & - & - & - & - \\
\hline $\begin{array}{l}\mathrm{EEDc}^{8} \\
D E E c^{8}\end{array}$ & $-1,9889$ & $<0,01$ & 0,9709 & 0,17 & 0,99 & $-1,99$ & - & - & - & - \\
\hline $\begin{array}{l}\text { EEDcj }^{9} \\
\text { DEEal }^{9}\end{array}$ & $-1,3278$ & $<0,01$ & 0,9379 & 0,01 & 0,96 & - & 0,7043 & $<0,01$ & 0,84 & $-29,57$ \\
\hline $\begin{array}{l}\mathrm{CNFDv}^{7} \\
D N F C f^{7}\end{array}$ & 2,0940 & 0,50 & 1,0260 & 0,83 & 0,92 & - & - & - & - & - \\
\hline $\begin{array}{l}\mathrm{CNFDc}^{8} \\
\mathrm{DNFCc^{8 }}\end{array}$ & $-0,7082$ & 0,49 & 0,9128 & 0,01 & 0,99 & - & 0,8979 & $<0,01$ & 1,00 & $-10,21$ \\
\hline $\begin{array}{l}\text { CNFDcj }^{9} \\
\text { DNFCa }^{9}\end{array}$ & 2,1440 & 0,24 & 0,8920 & 0,05 & 0,94 & - & 0,9430 & 0,06 & 0,98 & $-5,70$ \\
\hline
\end{tabular}

${ }^{1} \mathrm{Yi}={ }_{\hat{r n}}+\hat{\beta}_{\mathrm{i}} \mathrm{X}_{\mathrm{i}} ;{ }^{2} \mathrm{Y}_{\mathrm{i}}=\hat{\beta}_{\mathrm{i}} \mathrm{X}_{\mathrm{i}} ;{ }^{3} \mathrm{H}_{0}: \beta_{0}=0 ;{ }^{4} \mathrm{H}_{0}: \beta_{1}=1 ;{ }^{5} \mathrm{Em}$ pontos percentuais; ${ }^{6} \mathrm{Em}$ porcentagem; ${ }^{7}$ Alimentos volumosos.

${ }^{5}$ Percentage points; 6 In percentage; ${ }^{7}$ Forage. 
fração uniforme, fornecendo a mesma quantidade de energia independente do alimento; no entanto, as variações na digestibilidade do amido podem implicar em importantes exceções. Talvez diferentes equações sejam necessárias para estimar a energia disponível desta fração nas diferentes categorias de alimentos. O número de informações referente aos CNFD é relativamente pequeno para se ajustarem os valores dos coeficientes de digestibilidade médios.

\section{Conclusões}

Os valores de NDT obtidos a partir da equação proposta pelo NRC (2001) foram compatíveis com os valores observados de alimentos utilizados para alimentação animal nas condições brasileiras, principalmente para os alimentos concentrados. Entretanto, quando as equações que estimam a disponibilidade energética de cada fração do alimento foram analisadas isoladamente, verificaram-se algumas diferenças entre valores preditos e observados.

A equação que estima a FDND proposta pelo o NRC (2001) subestima a energia disponível da FDN de alimentos volumosos tropicais.

Para a fração nitrogenada dos alimentos, não foi constatada diferença entre os parâmetros avaliados, havendo boa correspondência entre valores preditos e observados.

O EED de alimentos volumosos e concentrados, utilizados em condições tropicais, é subestimado pela equação utilizada pelo NRC (2001), assim como os CNFD dos alimentos concentrados. Talvez diferentes equações sejam necessárias para estimar a energia disponível dessas frações, nas diferentes categorias de alimentos.

\section{Literatura Citada}

CONRAD, H.R.; WEISS, W.P.; ODWONGO, W.O. et al. Estimating net energy lactation from components of cell solubles and cell walls. Journal Dairy Science, v.67, p.427-436, 1984.

EDIONWEN, A.O.; OWEN, F.G. Relation of intake to digestibility of diets containing soy hulls and distillers dried grains. Journal Dairy Science, v.72, p.1786-1792, 1989.

NATIONAL RESEARCH COUNCIL - NRC. Nutrients requirements of dairy cattle. 4.ed. Washington, D.C.: 1971. 54p.

NATIONAL RESEARCH COUNCIL - NRC. Nutrient requirements of dairy cattle. 7.ed. Washington, D.C.: 2001. 381p. ROCHA JR., V.R.; VALADARES FILHO, S.C.; BORGES, A.M. et al. Determinação do valor energético dos alimentos para ruminantes. Revista Brasileira de Zootecnia, v.32, n.2, p.473-479, 2002.

VALADARES FILHO, S.C.; ROCHA JR., V.R.; CAPPELLE, E.R. Tabelas brasileiras de composição de alimentos para bovinos. Viçosa, MG: Universidade Federal de Viçosa, 2002. 297p.

VANDEHAAR, M. Efficiency of nutrient use and relationship to profitability on dairy farms. Journal Dairy Science, v.81, p.272-282, 1998.

WEISS, W.P. Predicting energy values of feed. in. symposium: prevailing concepts in energy utilization by ruminants. Journal Dairy Science, v.76, p.1802-1811, 1993.

WEISS, W.P. Estimating the availability energy content of feeds for dairy cattle. Symposium: energy availability. Journal Dairy Science, v.81, p.830-839, 1998.

WEISS, W.P.; CONRAD, H.R.; PIERRE, N.R. St. A theoretically-based model for predicting total digestible nutrient values of forages and concentrates. Animal Feed Science Technology, v.39, p.95-110, 1992.
Recebido em: 22/04/02 Aceito em: 16/09/02 